\title{
AVALIAÇÃO DA QUALIDADE EM LAN HOUSES ATRAVÉS DA ADAPTAÇÃO DO INSTRUMENTO SERVQUAL
}

\section{QUALITY ASSESSMENT IN LAN HOUSES THROUGH THE ADAPTATION OF THE SERVQUAL INSTRUMENT}

\author{
Tiago José Menezes Gonçalves* E-mail: tiagojmg@ita.br \\ Mischel Carmen Neyra Belderrain* E-mail: carmen@mec.ita.br \\ *Instituto Tecnológico de Aeronáutica , ITA, São José dos Campos, SP
}

\begin{abstract}
Resumo: Este trabalho foi realizado com o objetivo de desenvolver um questionário adaptado do Instrumento SERVQUAL para mensurar a Qualidade em Serviços prestados por Lan Houses. Além disso, pretendeu-se investigar a utilização da Análise de Quartis na priorização dos itens para a realização de ações corretivas para a melhoria da qualidade do serviço analisado. Visando estes objetivos, foi desenvolvida uma adaptação do Instrumento SERVQUAL com base na literatura e em entrevistas com gerentes de Lan Houses, a partir das quais foram extraídas as informações para sua adaptação ao objeto de estudo. Depois de desenvolvido, o instrumento foi aplicado e as implicações gerenciais de seu uso (em conjunto com a Análise de Quartis) foram discutidas com o gerente da Lan House, de onde foi obtido um feedback sobre a validade do modelo desenvolvido.
\end{abstract}

Palavras-chave: Qualidade em serviços. Análise de Quartis. SERVQUAL. Lan Houses. Modelo de Gap's.

\begin{abstract}
This work was conducted with the objective of developing an adapted questionnaire from the SERVQUAL instrument to measure the quality of services provided by Lan Houses. In addition, the use of analysis Quartiles was investigated in prioritizing the items to perform corrective actions to improve the quality of service analysis. For achieve this objectives, an adaptation of the SERVQUAL instrument was developed based on the literature and interviews with managers of Lan Houses, from where information was extracted for its adaptation to the object of study. Once developed, the instrument was used and the managerial implications of its use (in conjunction with the Quartiles Analysis) were discussed with the Lan House's manager, from where feedback was obtained for the validity of the model developed.
\end{abstract}

Keywords: Service quality. Analysis of Quartiles. SERVQUAL. Lan Houses. Gap's model.

\section{INTRODUÇÃO}

Nos últimos anos, com o avanço da globalização e a intensificação da competição, muitas organizações têm procurado maneiras de se diferenciar no mercado, atingindo uma parcela maior de consumidores. 
De acordo com Kotler e Armstrong (1998), uma das principais maneiras de uma empresa se diferenciar no mercado é fornecendo serviços de qualidade. A entrega de serviços com alto nível de qualidade provê às empresas uma maior aquisição e retenção de clientes (FERGUSON; ZAWACKI, 1993), culminando em vantagem competitiva. Por sua vez, a vantagem competitiva surge quando uma empresa consegue criar para seus clientes um valor maior que o custo de fabricação/prestação do serviço (PORTER, 1999). Neste sentido, conclui-se que as ações tomadas em prol da melhoria da qualidade são diretamente relacionadas com a geração de valor para o cliente, com uma maior qualidade significando maior valor ao produto ou serviço prestado.

No contexto da prestação de serviços, a qualidade é um dos conceitos mais discutidos na literatura, principalmente devido a não existência de um consenso sobre sua definição ou ao modo mais adequado de realizar sua medição (SCHNEIDER; WHITE, 2004).

Muitos pesquisadores vêm trabalhando no desenvolvimento de técnicas e modelos para realizar a medição da qualidade em serviços. Dentre os modelos existentes, o modelo de Gap's (PARASURAMAN; ZEITHAML; BERRY, 1985, 1988, 1991; PARASURAMAN; BERRY; ZEITHAML, 1990, 1991) é um dos mais difundidos, tendo sido implementado pelos mesmos autores através do instrumento SERVQUAL. Este modelo considera que a qualidade em serviços é uma função da diferença entre a percepção do usuário a respeito do desempenho do serviço e a sua expectativa sobre o mesmo.

Tendo em vista estas considerações, este trabalho tem como objetivo desenvolver um formulário adaptado do Instrumento SERVQUAL para mensurar a qualidade em serviços prestados por Lan Houses. Além disso, pretende-se investigar a utilização da Análise de Quartis (FREITAS; MANHÃES; COZENDEY, 2006; GONÇALVES; FREITAS, 2010a) na priorização dos itens para a realização de ações corretivas para a melhoria da qualidade.

De maneira breve, este artigo está estruturado na seguinte forma: a seção 2 trata da fundamentação teórica relacionada ao conceito de qualidade em serviços, do 
Modelo de Gap's e do Instrumento SERVQUAL, e da descrição e relevância do conceito de Lan House; a seção 3 descreve a metodologia utilizada neste trabalho; a seção 4 apresenta os resultados obtidos, com a identificação dos itens críticos e sugestão de ações de melhoria; por fim, a seção 5 apresenta considerações finais do presente trabalho.

\section{FUNDAMENTAÇÃO TEÓRICA}

\subsection{Qualidade em serviços}

De acordo com Parasuraman, Zeithaml e Berry (1985), as pesquisas sobre a mensuração da qualidade em serviços iniciaram-se após a preocupação com o aprimoramento da qualidade em produtos. Os mesmos autores definem a qualidade de um serviço como o grau em ele atende as expectativas de um cliente, e afirmam que ela é mais difícil de ser mensurada do que a qualidade de bens físicos devido às características dos serviços, que são compostos em grande parte por experiências.

Neste sentido, Parasuraman, Zeithaml e Berry (1988) apresentaram três características dos serviços, conforme abaixo:

— Simultaneidade: serviços são produzidos e consumidos simultaneamente;

—Intangibilidade: serviços representam um produto não físico, não podendo ser transportados e/ou armazenados.

- Heterogeneidade: a prestação de determinado serviço varia dependendo de quem o executa e de onde é prestado, mudando de cliente para cliente e de um momento para o outro.

As características listadas acima implicam em uma série de consequências, entre as quais convém mencionar que o cliente é afetado imediatamente por qualquer falha no sistema de prestação (já que é um elemento presente neste), o que torna difícil ou quase impossível detectar e corrigir eventuais falhas antes que elas ocorram e afetem o cliente. Além disso, não é possível criar estoques de segurança para absorver as variações na demanda e a heterogeneidade dos serviços dificulta ou mesmo 
impossibilita sua padronização, já que o serviço é realizado com diferentes performances em momentos diferentes.

De acordo com Lovelock e Wright (2003), os clientes julgam a qualidade de um serviço a partir de um padrão interno existente antes de sua experiência com o serviço prestado. Este padrão é formado com base em suas experiências anteriores e é a base para a formação de suas expectativas.

Essas expectativas correspondem aos desejos dos clientes em relação a como a prestação de um serviço deveria ser (PARASURAMAN; ZEITHAML; BERRY, 1988). Segundo Grönros (1993), os consumidores escolhem prestadores de serviços através da comparação entre a percepção que tem do serviço e as expectativas acerca do mesmo.

Esta última afirmação está baseada no paradigma da desconfirmação proposto por Oliver (1981), que afirma que a qualidade de um serviço é uma função da diferença entre o serviço percebido e o serviço esperado, sendo ratificada por diversos autores (PARASURAMAN; ZEITHAML; BERRY, 1985; BROWN; SWARTZ, 1989; CARMAN, 1990). Neste sentido, podem ocorrer três tipos de desconfirmação:

- Desconfirmação negativa: quando o desempenho de um serviço é pior que as expectativas;

- Desconfirmação positiva: quando o serviço apresenta um desempenho melhor que as expectativas;

— Desconfirmação zero: quando o desempenho do serviço é igual às expectativas.

A idéia anterior é a base para o Modelo de Gap's e o Instrumento SERVQUAL (PARASURAMAN; ZEITHAML; BERRY, 1985, 1988, 1991; PARASURAMAN; BERRY; ZEITHAML, 1990, 1991) para a Avaliação da Qualidade em Serviços, os quais são apresentados na próxima seção.

\subsection{Modelo de Gap's e Instrumento SERVQUAL}

Baseado no modelo de satisfação de Oliver (1980), Parasuraman, Zeithaml e Berry (1985) propuseram um modelo para medição da qualidade em serviços, 
denominado Modelo de Gap's, onde a qualidade de um serviço é uma função da diferença entre a expectativa do cliente e o desempenho do serviço. Dessa forma, a avaliação $Q_{i}$ de um serviço em relação a uma dimensão $i$ da qualidade em serviços é realizada pela diferença entre a expectativa dos clientes $E_{i}$ e a percepção $D_{i}$ dos mesmos sobre o desempenho do serviço, conforme descrito na equação 1.

$$
Q_{i}=P_{i}-E_{i}
$$

Essa diferença (ou Gap) entre a expectativa e a percepção de desempenho do serviço seria uma medida da qualidade de um serviço em relação à dimensão $i$. Essas dimensões são características genéricas dos serviços que, ao serem avaliadas sob a perspectiva dos clientes e em conjunto, resultariam na avaliação da qualidade do serviço como um todo. Esse modelo destaca as cinco lacunas ou Gap's da qualidade na prestação do serviço, conforme apresentado na Fig. 1.

Os cincos Gap's ou lacunas do modelo são discutidos a seguir:

- Gap 1: Diferença entre as expectativas do usuário e a percepção gerencial: trata-se da lacuna existente entre a percepção dos gestores e as reais expectativas dos consumidores. Resulta de uma compreensão inadequada por parte dos gestores sobre como os clientes formam suas expectativas, devido a:

- Falta de informações vindas da linha de frente para os gestores;

- Interpretação equívoca das expectativas dos clientes;

- Perda de informações pelos níveis hierárquicos;

- Inexistência ou imprecisão nas informações obtidas de análises de demanda e/ou pesquisas de mercado.

- Gap 2: Diferença entre a percepção gerencial das expectativas dos usuários e as especificações da qualidade do serviço: Trata-se da não inclusão, pelos gestores, de todos os elementos capazes de atender as expectativas dos clientes nas especificações da qualidade do serviço, resultando num entendimento incorreto das expectativas dos clientes. As principais causas desta falha são:

- Erros na elaboração das especificações e de planejamento; Revista Produção Online, Florianópolis, SC, v.12, n. 1, p. 248-268, jan./mar. 2012. 
- Falta de compromisso dos gestores;

- Falta do estabelecimento de metas claras.

- Gap 3: Diferença entre as especificações da qualidade do serviço e a prestação do serviço: Mesmo que as especificações do serviço sejam adequadas, os funcionários podem cometer erros e comprometer a qualidade final do serviço. Assim, esse Gap trata da diferença entre os procedimentos especificados e o desempenho real na prestação do serviço.

Figura 1 - Os 5 Gap's da Qualidade em Serviços

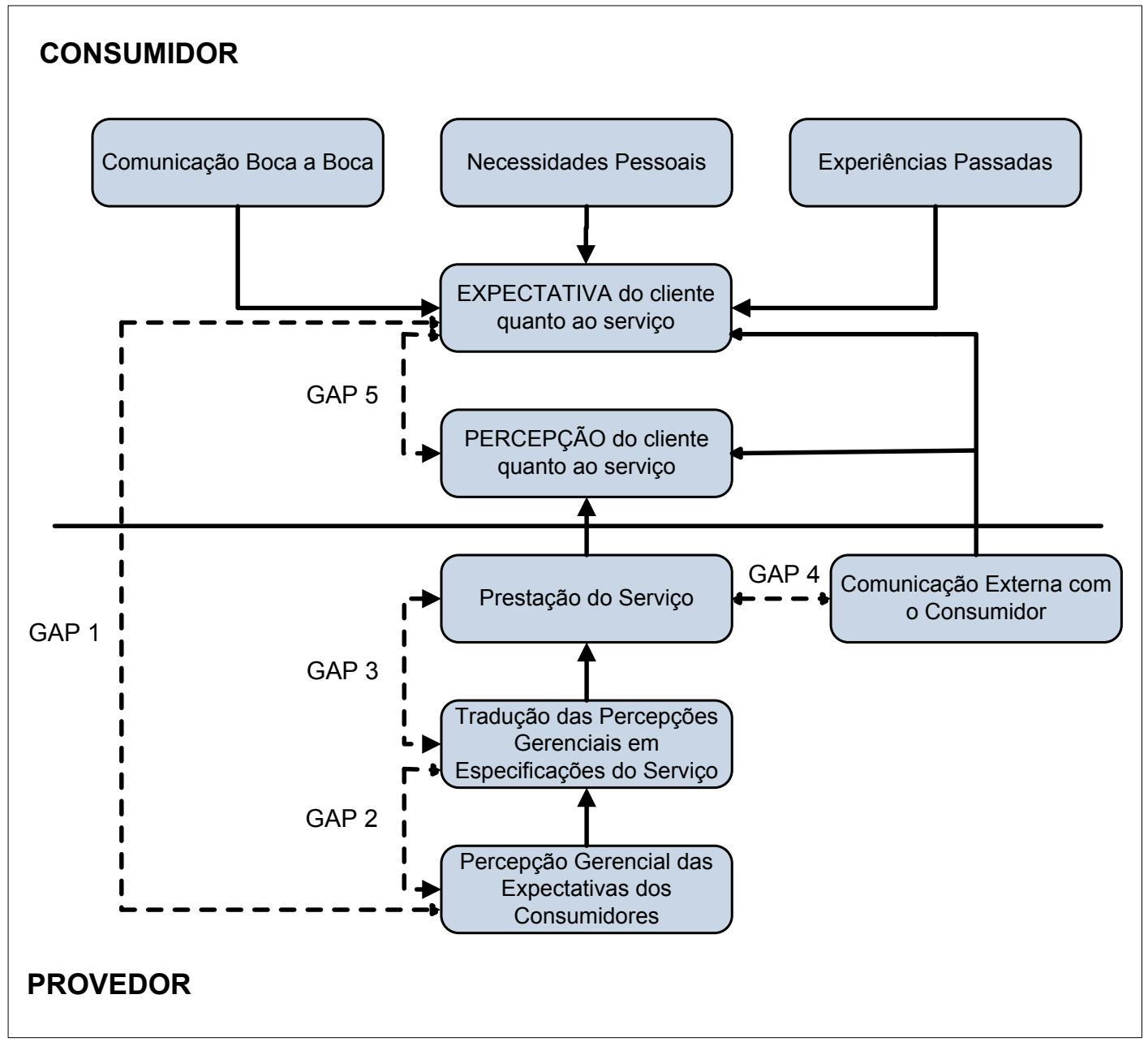

Fonte: Parasuraman; Zeithaml; Berry (1985).

- Gap 4: Diferença entre a percepção do serviço e as comunicações externas com o usuário: Trata-se da diferença entre o serviço prometido e o serviço prestado ao Revista Produção Online, Florianópolis, SC, v.12, n. 1, p. 248-268, jan./mar. 2012. 
usuário. Através da propagada e/ou diversas outras formas de comunicação, são realizadas promessas para o cliente sobre o nível de desempenho na prestação do serviço. Por sua vez, essas promessas aumentam as expectativas dos clientes e, caso não forem cumpridas, geram insatisfação nos clientes.

- Gap 5: Lacuna entre o serviço esperado e o serviço percebido: Trata-se do resultado dos Gap's anteriores, e ocorre quando um ou mais dos anteriores ocorrem. Esse Gap, que corresponde à diferença entre o serviço esperado e a qualidade percebida pelo consumidor, é uma função dos Gap's anteriores:

$$
\text { Gap } 5 \text { = f(Gap 1, Gap 2, Gap 3, Gap 4) }
$$

A partir do Modelo de Gap's, Parasuraman, Zeithaml e Berry (1988) desenvolveram o Instrumento SERVQUAL para a avaliação da qualidade percebida pelos clientes. Este instrumento, também conhecido como modelo, ferramenta ou escala SERVQUAL, utiliza o Gap 5 do Modelo de Gap's para avaliar a qualidade percebida pelos clientes, através de 22 itens que abordam critérios relativos a cinco dimensões da qualidade, que são:

- Confiabilidade: capacidade para realizar um serviço de forma confiável;

- Presteza: capacidade de prover atendimento imediato e ajudar o cliente;

- Segurança: capacidade de transmitir segurança, confiança, cortesia e conhecimento;

- Empatia: capacidade de fornecer atenção individualizada aos clientes;

- Aspectos tangíveis: adequação de equipamentos, instalações, pessoal envolvido e materiais.

O emprego deste instrumento é realizado com a captação das expectativas e percepções dos clientes para cada item, através da utilização de uma Escala Likert de 7 pontos com os extremos marcados com "Discordo Totalmente" e "Concordo Totalmente". Após a coleta de dados, é obtida uma pontuação final através da diferença entre as percepções e as expectativas (Gap $5=P-E$ ). Uma pontuação positiva indica que o serviço prestado está superando a expectativa, o que gera satisfação para o cliente. Uma pontuação negativa indica que o desempenho do serviço está abaixo do Revista Produção Online, Florianópolis, SC, v.12, n. 1, p. 248-268, jan./mar. 2012. 
esperado, revelando quais critérios geram insatisfação para os clientes e possibilitando a intervenção dos gestores para a melhoria da qualidade.

Ainda segundo Parasuraman, Zeithaml e Berry (1988), a ferramenta SERVQUAL pode ser utilizada por uma grande variedade de empresas de serviços, sendo necessária a adaptação do instrumento de pesquisa ajustando os 22 itens ao contexto específico do serviço avaliado.

\subsection{Descrição e Relevância das Lan Houses}

De acordo com Torreta (2009), o conceito de Lan House teve início na Coréia em 1996, chegando ao Brasil em 1998. Segundo Costa et al. (2005), no termo Lan House, a palavra Lan tem origem do inglês Local Area Network, que representa uma rede de computadores, sendo oriundo do fato que uma Lan House é constituída de diversos computadores conectados em rede à Internet de banda larga.

Segundo Magalhães e Garcia (2008), as Lan Houses surgem em meio ao desejo de conexão local e global que representa uma nova forma de mobilização, sendo o objetivo dos clientes a navegação, a interatividade e a simulação através do uso de equipamentos digitais.

A estrutura de uma Lan House caracteriza-se por um ambiente tecnológico onde os clientes se divertem com jogos e/ou navegando pela Internet, geralmente em um espaço contendo ar condicionado e cadeiras ergonômicas. Em geral, as Lan Houses utilizam um software que controla o acesso a todos os computadores, através do qual é possível obter estatísticas de utilização, da receita gerada por cada máquina e sobre os horários de maior utilização dos equipamentos. Isto possibilita a extração de dados importantes para a gestão dos negócios, possibilitando, por exemplo, criar promoções nos horários de baixa utilização dos equipamentos e o redimensionamento da disponibilidade destes para atender os clientes nos picos de utilização (COSTA et al., 2005).

Borges (2009) descreve as Lan Houses como estabelecimentos comerciais que possibilitam o acesso à Internet a partir de um preço cobrado por tempo de utilização. 
Segundo este autor, em vista do crescimento da penetração do computador pessoal e da Internet domiciliar e da oferta de rede Wi-Fi em centros comerciais como shoppings, lanchonetes, etc., as Lan Houses deixaram de ser lucrativas nas zonas centrais das cidades e foram migrando para as periferias, indo ao encontro do apoio recebido das políticas públicas e privadas para promover maior cobertura do acesso à Internet nessas regiões.

Segundo Costa et al. (2005) o público de uma Lan House é variado, sendo que à noite e de madrugada os adultos dominam o local para acessar a Internet, consultar emails e entrar em salas de bate-papo, porém durante 0 dia o público é predominantemente composto por jovens, que têm preferência por serviços de jogos em rede.

Por fim, Borges (2009) ressalta a relevância das Lan Houses nas periferias e destaca seu papel social, atuando como ponto de encontro, conversa, contato, relacionamento e sociabilidade de grupos jovens.

\section{METODOLOGIA}

Inicialmente foi realizado o desenvolvimento do questionário, onde o Instrumento SERVQUAL foi adaptado para a avaliação da qualidade em serviços prestados em Lan Houses. Essa adaptação foi baseada em entrevistas com especialistas do setor e em trabalhos acadêmicos de áreas correlatas, principalmente aqueles realizados por Gonçalves e Freitas (2009, 2010a, 2010b).

O questionário aplicado contém 16 itens e avalia 6 dimensões da qualidade, que são: aspectos tangíveis, equipamentos, confiabilidade, presteza, segurança e empatia (ver apêndice 1). Com exceção da dimensão "equipamentos", que é proposta neste trabalho, todas as demais são adaptadas do instrumento SERVQUAL. A dimensão equipamentos foi proposta devido às características peculiares dos serviços prestados por Lan Houses, onde o cliente passa a maior parte do tempo em que está inserido no sistema de prestação de serviços interagindo com os equipamentos disponibilizados, o que torna o desempenho desses equipamentos um fator central no processo de 
prestação do serviço. Além disso, com o objetivo de obter uma amostra de maior tamanho, procurou-se utilizar um questionário de tamanho reduzido visando não desmotivar o cliente em respondê-lo. Para reduzir o tamanho do questionário, foram enfatizados os itens do instrumento SERVQUAL identificados como de maior importância para o objeto de estudo pelo o gestor da Lan House, adaptando-o ao objeto de estudo. Em relação ao formulário de respostas, o questionário desenvolvido contém um formulário de resposta para Expectativa ( $E$ ) e outro para Desempenho ( $P$ ), onde são utilizadas escalas do tipo Likert com cinco pontos cada. Estas escalas buscam captar o Grau de Concordância/Discordância dos usuários em relação às sentenças afirmativas relativas aos critérios considerados, variando de "Discordo Totalmente", para 1, até "Concordo Totalmente", para 5.

Depois de finalizado o desenvolvimento do questionário, duzentas cópias impressas foram entregues a uma funcionária da Lan House em estudo, a qual foi instruída e encarregada de sua aplicação. Cabe ressaltar que o procedimento de utilização da Lan House inicia-se com o cadastramento do cliente quando este utiliza o serviço pela primeira vez, sendo que somente depois de executado este procedimento um computador é liberado para sua utilização. Este procedimento permitiu saber de antemão quantos usuários tinham utilizado o serviço anteriormente ao menos uma vez nos últimos três meses. Com isso objetivou-se tornar a amostra mais homogênea e evitar clientes que não tinham frequentado anteriormente o serviço e, portanto, estavam menos preparados para responder aos itens do questionário.

A aplicação do questionário foi realizada durante um período de três semanas, durante o qual foi obtida uma amostra de 143 respondentes, que constituíam $45 \%$ dos clientes cadastrados na Lan House (que utilizaram o serviço anteriormente ao menos uma vez). Os questionários foram entregues aos respondentes ao final da utilização do serviço, sendo estes solicitados a preencher os questionários quando se dirigiam ao caixa para realizar o pagamento. Ressalta-se que o objeto de estudo deste trabalho apresenta algumas características especiais que possibilitaram obter uma amostra maior do que o esperado. Por se tratar de uma Lan House que é a única em uma cidade pequena, esta não possui concorrentes e atende a toda população da cidade. 
Este fato também faz com que os clientes da mesma sejam menos eventuais do que aqueles normalmente encontrados no setor de serviços devido à unicidade do serviço na cidade em que é prestado.

Em relação à análise, depois de coletados os dados foram analisados com métodos estatísticos de apoio à decisão, de onde foram obtidos indicadores da qualidade dos diversos critérios analisados, bem como a ordem de priorização dos recursos a serem aplicados na melhoria da qualidade dos serviços segundo cada critério.

Por fim, realizou-se uma análise crítica junto ao gerente responsável pelo serviço, onde foram apresentados os resultados das análises e realizadas as propostas de melhoria para serem contempladas pelo tomador de decisão, o que possibilitou analisar a validade dos resultados obtidos e obter um feedback para a conclusão do trabalho.

\section{RESULTADOS}

A tabela 1 apresenta os valores da Expectativa Média $(\bar{E})$, da Percepção Média $(\bar{P})$ e do Gap Médio $(\bar{G})$ segundo cada critério avaliado. Geralmente, os itens considerados mais críticos são aqueles que apresentam maiores Gap's negativos.

A avaliação da qualidade dos serviços prestados pela Lan House estudada trouxe evidências através da análise do Gap médio de que a mesma tem satisfeito seus clientes em relação às dimensões "aspectos tangíveis" $(0,09)$, "presteza" $(0,02)$, "segurança" $(0,30)$ e "empatia" $(0,12)$. Os critérios que são percebidos como os de maior qualidade para os clientes são aqueles referentes ao item 14 ("Existe suporte adequado para o uso dos equipamentos"), ao item 3 ("O ambiente é iluminado"), ao item 4 ("Os funcionários são bem vestidos e asseados") e ao item 16 ("O horário de funcionamento é conveniente"). Por outro lado, a avaliação realizada também evidenciou que a empresa estudada apresenta um serviço com baixa qualidade segundo a percepção de seus clientes em relação às dimensões "equipamentos" ($0,98)$ e "confiabilidade" $(-0,40)$. 
Para identificar aqueles itens que comprometem a percepção do cliente sobre a qualidade do serviço prestado foi utilizada a Análise de Quartis, proposta em Freitas, Manhães e Cozendey (2006).

A proposta da Análise de Quartis é que, diferentemente do contexto estatístico, onde as medidas de tendência central denominadas quartis são utilizadas meramente como indicadores de como os dados estão distribuídos, no contexto da avaliação da qualidade em serviços essas medidas são utilizadas para classificar/priorizar itens cujos valores se situam nas quatro classes geradas utilizando-se os valores dos quartis como divisores de classe. Cada classe contêm $25 \%$ dos itens cada uma, sendo os itens atribuídos a uma das quatro classes (Prioridade Crítica, Prioridade Alta, Prioridade Moderada e Prioridade Baixa) conforme sua prioridade.

Tabela 1 - Dimensões da qualidade

\begin{tabular}{lccc}
\hline \multicolumn{1}{c}{ Dimensões e Itens } & Percepção & $\begin{array}{c}\text { Expectativa } \\
(\bar{E})\end{array}$ & $\begin{array}{c}G A P \\
(\bar{G})\end{array}$ \\
\hline A. Aspectos Tangíveis & 4,50 & $\mathbf{4 , 4 1}$ & $\mathbf{0 , 0 9}$ \\
$1 \quad$ O ambiente é limpo. & 4,20 & 4,81 & $-0,61$ \\
$2 \quad$ O ambiente é ventilado. & 4,80 & 4,65 & 0,15 \\
$3 \quad$ O ambiente é iluminado. & 4,65 & 4,22 & 0,43 \\
$4 \quad$ Os funcionários são bem vestidos e asseados. & 4,36 & 3,95 & 0,41 \\
B. Equipamentos & 3,66 & $\mathbf{4 , 6 4}$ & $-\mathbf{0 , 9 8}$ \\
$5 \quad$ Velocidade de acesso à Internet é alta. & 3,21 & 4,89 & $-1,68$ \\
$6 \quad$ Desempenho dos computadores é adequado. & 3,58 & 4,77 & $-1,19$ \\
$7 \quad$ Softwares instalados são adequados. & 4,20 & 4,26 & $-0,06$ \\
C. Confiabilidade & $\mathbf{3 , 9 3}$ & $\mathbf{4 , 3 3}$ & $\mathbf{- 0 , 4 0}$ \\
$8 \quad$ Os computadores funcionam corretamente. & 4,43 & 4,58 & $-0,15$ \\
$9 \quad$ Existe segurança contra a transmissão de malwares. & 3,12 & 4,33 & $-1,21$ \\
$10 \quad$ Funciona nos horários divulgados. & 4,23 & 4,09 & 0,14 \\
D. Presteza & 4,28 & $\mathbf{4 , 2 6}$ & $\mathbf{0 , 0 2}$ \\
$11 \quad$ O serviço é fornecido assim que solicitado. & 4,01 & 4,15 & $-0,14$ \\
12 Os funcionários são dispostos a ajudar os clientes. & 4,56 & 4,36 & 0,20 \\
E. Segurança & $\mathbf{4 , 4 8}$ & $\mathbf{4 , 1 8}$ & $\mathbf{0 , 3 0}$ \\
$13 \quad$ Os Funcionários são educados. & 4,62 & 4,60 & 0,02 \\
$14 \quad$ Existe suporte adequado para o uso dos equipamentos. & 4,34 & 3,77 & 0,57 \\
F. Empatia & $\mathbf{4 , 3 2}$ & $\mathbf{4 , 2 0}$ & $\mathbf{0 , 1 2}$ \\
$15 \quad$ Os funcionários fornecem atenção individual. & 4,01 & 4,12 & $-0,11$ \\
$16 \quad$ O horário de funcionamento é conveniente. & 4,63 & 4,28 & 0,35 \\
\hline Fon & &
\end{tabular}

Fonte: Elaborado pelos autores

Revista Produção Online, Florianópolis, SC, v.12, n. 1, p. 248-268, jan./mar. 2012. 
Em relação às limitações da Análise de Quartis, menciona-se que o número de classes e de itens por classe considerados por esta análise são fixos e não podem ser alterados conforme as necessidades dos pesquisadores, o que reduz a flexibilidade do método. Por outro lado, embora esses fatores possam ser vistos como limitações, eles constituem a base que sustenta a simplicidade na utilização do método, que consiste em um de seus principais pontos fortes.

Comparada a outros métodos já consolidados na literatura, como o IPA (Importance Performance Analysis), a Análise de Quartis apresenta como vantagem a não necessidade de coletar a importância referente a cada item do questionário. Este fator, além de prover uma análise mais simples dos dados, resulta em um maior tamanho da amostra, já que há um aumento de trabalho no preenchimento do questionário pelo cliente quando existe a presença de um formulário extra para a coleta das importâncias de cada item no caso do IPA, o que consiste em um aspecto desmotivante para o respondente.

Além disso, esta análise vem sendo empregada com sucesso por diversos autores (FREITAS; COZENDEY, 2008; FREITAS; MORAIS, 2008a, 2008b; GONÇALVES; FREITAS, 2009, 2010a, 2010b), o que motivou sua utilização neste trabalho. Em relação aos resultados obtidos, estes podem ser observados nas tabelas 2,3 e 4 .

Tabela 2 - Priorização dos Itens através da Análise de Quartis $(\bar{P})$

\begin{tabular}{|c|c|c|c|c|c|c|c|c|c|c|c|c|c|c|c|}
\hline \multicolumn{16}{|c|}{ Classificação de prioridade dos itens - Percepções } \\
\hline $\begin{array}{c}I_{9} \\
312\end{array}$ & $\begin{array}{c}I_{5} \\
321\end{array}$ & $\begin{array}{c}I_{6} \\
358\end{array}$ & $\begin{array}{c}l_{15} \\
401\end{array}$ & $\begin{array}{c}l_{11} \\
4.01\end{array}$ & $\begin{array}{c}I_{7} \\
4.20\end{array}$ & $\begin{array}{c}\mathrm{l}_{1} \\
4.20\end{array}$ & $\begin{array}{c}\mathrm{l}_{10} \\
4.23\end{array}$ & $\begin{array}{c}\mathrm{I}_{14} \\
4.34\end{array}$ & $\begin{array}{c}\mathrm{I}_{4} \\
436\end{array}$ & $\begin{array}{c}\mathrm{I}_{8} \\
4.43\end{array}$ & $\begin{array}{c}\mathrm{I}_{12} \\
4.56\end{array}$ & $\begin{array}{c}\mathrm{I}_{13} \\
4.62\end{array}$ & $\begin{array}{c}I_{16} \\
463\end{array}$ & $\begin{array}{c}\mathrm{I}_{3} \\
4.65\end{array}$ & $\begin{array}{c}\mathrm{I}_{2} \\
4.80\end{array}$ \\
\hline \multicolumn{6}{|c|}{$1^{\circ}$ Quartil $=4,01$} & \multicolumn{4}{|c|}{$2^{\circ}$ Quartil $=4,28$} & \multicolumn{5}{|c|}{$3^{\circ}$ Quartil $=4,60$} & \\
\hline \multicolumn{3}{|c|}{ Prioridades: } & \multicolumn{2}{|c|}{ Crítica } & & \multicolumn{3}{|c|}{ Alta } & & \multicolumn{3}{|c|}{ Moderada } & \multicolumn{3}{|c|}{ Baixa } \\
\hline
\end{tabular}

Fonte: Elaborado pelos autores

Tabela 3 - Priorização dos Itens através da Análise de Quartis $(\bar{E})$

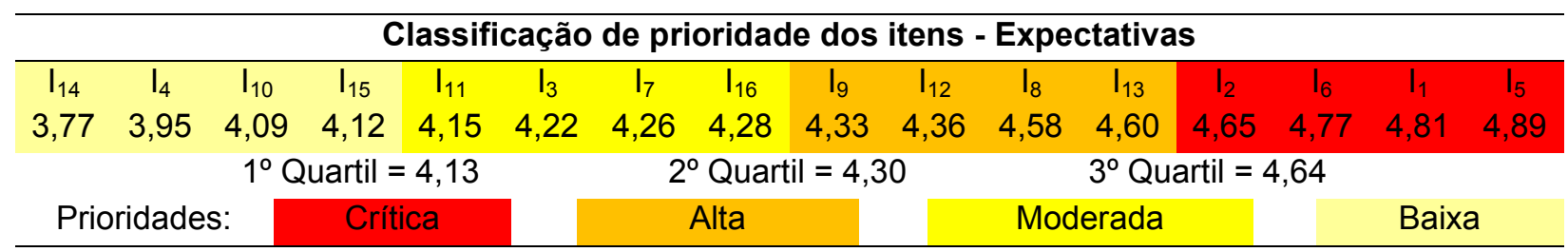

Fonte: Elaborado pelos autores 
Tabela 4 - Priorização dos Itens através da Análise de Quartis $(\bar{G})$

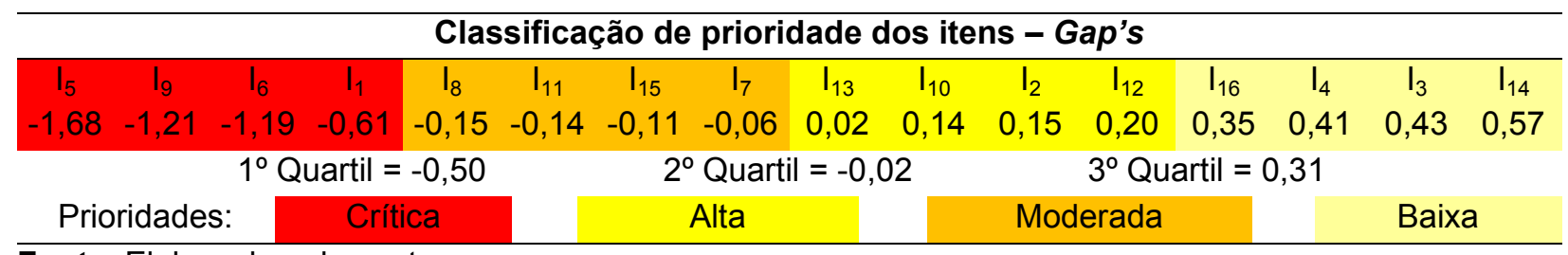

Fonte: Elaborado pelos autores

Como pode ser observados na tabela 3 , os itens $I_{5}, I_{1}, I_{6}$ e $I_{2}$ são aqueles que apresentam as maiores expectativas segundo a percepção dos clientes. Esses itens pertencem às dimensões "Aspectos Tangíveis" $\left(I_{1}\right.$ e $\left.I_{2}\right)$ e "Equipamentos" $\left(I_{5}\right.$ e $\left.I_{6}\right)$, e referem-se respectivamente à limpeza e ventilação do ambiente, a velocidade de acesso a Internet e ao desempenho dos computadores.

Com o objetivo de identificar as prováveis causas que evidenciaram os itens críticos na análise dos Gap's (ver tabela 4) e formular propostas para a melhoria do serviço, foi realizada uma reunião com o gerente da Lan House e os autores deste trabalho. A seguir apresenta-se sucintamente os resultados da discussão sobre cada item considerado como de Prioridade Crítica:

—Item 5 (Velocidade de acesso à Internet é alta): De acordo com o gerente responsável pela Lan House estudada, os problemas ocasionados pela velocidade baixa da Internet têm como origem o serviço inadequado prestado pela empresa que provê o acesso a Internet. Segundo o mesmo, a velocidade do pacote de dados contratado nem sempre é atendida pela empresa, ficando muitas vezes bem abaixo do valor nominal contratado, principalmente nos horários de pico ocorridos durante a tarde. Além disso, o servidor sai do ar com certa freqüência, principalmente durante tempestades com a ocorrência de raios. O gerente afirma que já entrou em contato com a empresa, a qual respondeu que está aumentando sua capacidade para conseguir contornar os problemas decorrentes dos horários de pico. Informou também que desliga os aparelhos durante a ocorrência de raios para preservá-los de descargas elétricas que possam danificá-los.

—Item 9 (Existe segurança contra a transmissão de malwares): Em relação à segurança na utilização dos equipamentos, o gerente afirma que a única providência 
que a Lan House realiza no sentido de evitar a contaminação de seus equipamentos por malwares trata-se da instalação e atualização periódica de softwares antivírus em seus computadores. Como ação corretiva foi sugerida ao gerente à realização de configurações para o bloqueio da instalação de softwares pelos usuários e a instalação de um firewall para a proteção de sua intranet.

— Item 6 (Desempenho dos computadores é adequado): Em relação a este item, o gerente da Lan House afirma que a mesma possui alguns computadores com muitos anos de uso, sendo que estes equipamentos ainda não foram substituídos por falta de verba. Devido a este fato, muitos computadores apresentam desempenho inadequado devido à desatualização tecnológica, o que justifica a criticidade deste item. Neste sentido, foram feitas recomendações ao gerente sobre a realização de upgrades nos componentes dos equipamentos considerados ultrapassados, em vista desta prática ser menos dispendiosa que a aquisição do equipamento completo. Além disso, recomendou-se a venda de equipamentos já obsoletos para o uso pela Lan House como fonte para a obtenção de verba para a compra de novos equipamentos.

—Item 1 (O ambiente é limpo): No que se refere à limpeza no ambiente da Lan House, o gerente responsável afirma que existe somente um funcionário que é encarregado tanto pelo atendimento aos clientes quanto pela limpeza do ambiente e demais atividades. Segundo o gerente, este funcionário muitas vezes fica sobrecarregado de trabalho e não consegue realizar algumas de suas tarefas, o que justifica a criticidade deste item. Almejando solucionar o problema, foram feitas recomendações para o gerente da Lan House no sentido de contratar outro atendente para os horários de pico do estabelecimento, proporcionando maior tempo útil para a limpeza do mesmo.

\section{CONSIDERAÇÕES FINAIS}

Este artigo teve como objetivo propor um modelo para a avaliação da qualidade em serviços prestados por Lan Houses baseado no Instrumento SERVQUAL. Além 
disso, buscou-se avaliar a utilização da Análise de Quartis na priorização dos itens para a realização de ações corretivas para a melhoria da qualidade.

No que se refere ao modelo desenvolvido, o gerente da Lan House estudada afirmou que ficou satisfeito com os resultados obtidos. Segundo o mesmo, várias das informações sobre as percepções dos clientes vieram ao encontro de aspectos já identificados intuitivamente por ele, referindo-se aos problemas com a velocidade da Internet e ao desempenho dos computadores. Além disso, o modelo evidenciou necessidades de melhorias antes não cogitadas pelo decisor, referentes à limpeza do ambiente e a segurança contra a transmissão de malwares. A utilização do modelo desenvolvido permitiu captar as percepções dos clientes de forma a fornecer diversas informações gerenciais que culminaram em propostas importantes para a melhoria da qualidade do serviço analisado.

Em relação à Análise de Quartis, esta permitiu identificar os itens de maior criticidade/urgência, ou seja, aqueles que devem ser priorizados na realização de ações para melhoria na qualidade dos serviços prestados pela Lan House. A Análise de Quartis mostrou-se como uma ferramenta simples e prática no auxílio à avaliação da qualidade em serviços. A discussão dos resultados desta análise com o gestor da Lan House possibilitou estabelecer ações corretivas visando melhorar a percepção da qualidade do serviço pelos clientes. Neste sentido, a Análise de Quartis mostrou-se como uma ferramenta simples e prática para a priorização dos itens na avaliação da qualidade em serviços.

Cabe destacar que o resultado desta pesquisa foi informado a todos os respondentes por e-mail, onde foram relatados os itens de prioridade crítica identificados e as possíveis ações para melhoria da qualidade a serem realizadas. Além disso, foi disponibilizado um e-mail para o envio de sugestões pelos clientes, onde se espera captar dos usuários ideias novas para o aperfeiçoamento das ações de melhoria propostas. Considera-se esta atitude muito importante, pois busca conscientizar o respondente de que o seu trabalho em responder a pesquisa não foi em vão e, mais ainda, contribuirão para a realização de ações de melhoria que realmente serão implementadas. 
Por fim, ressalta-se que os resultados apresentados aqui retratam apenas a avaliação da qualidade dos serviços prestados pela Lan House em um período específico, e que a continuidade das avaliações em períodos subseqüentes poderá fornecer resultados mais conclusivos, contribuindo para a verificação das ações de melhoria aqui propostas.

\section{REFERÊNCIAS}

BORGES, F. M. Sociabilidade nas Lan Houses das periferias. Ponto-e-Vírgula, v. 6, p. 218-234, 2009.

BROWN, S. W.; SWARTZ, T. A. A gap analysis of professional service quality. Journal of Marketing, Chicago, v. 53, n. 2, p. 92-98, apr. 1989.

CARMAN, J. M. Consumer perceptions of service quality: An assessment of the SERVQUAL dimensions. Journal of Retailing, Greenwich, v. 66, n. 1, p. 33-55, spring 1990.

COSTA, A. B.; RIBEIRO, B.; ALMEIDA, G. H. M.; PRETA, J. O. C.; SILVA, V. B. A gestão de planejamento estratégico na gestão de pequenas e médias empresas. Belo Horizonte, MG: FEAD, 2005. Originalmente apresentado como Monografia de Conclusão de Curso no MBA em Gestão de Negócios da FEAD - Minas, Faculdade de Estudos Administrativos de Minas Gerais, 2005.

FREGUSON, J. M.; ZAWACKI, R. A. Service quality: a critical success factor for IS organizations. Information Strategy: The Executive's Journal, v. 9, n. 2, p. 24-30, 1993.

FREITAS, A. L. P.; BOLSANELO, F. M. C.; VIANA, N. R. N. G. Avaliação da qualidade de serviços de uma biblioteca universitária: um estudo de caso utilizando o modelo SERVQUAL. Revista Ciência da Informação, Brasília, v. 37, n. 3, p. 88-102, set-dez. 2008.

FREITAS, A. L. P.; COZENDEY, M. I. Um modelo SERVPERF para avaliação de serviços hospitalares. In: ENCONTRO NACIONAL DE ENGENHARIA DE PRODUÇÃO, 28., 2008, Rio de Janeiro. Anais... Rio de Janeiro: Associação Brasileira de Engenharia de Produção, 2008. p. 1-13.

FREITAS, A. L. P.; MANHÃES, N. R. C.; COZENDEY, M. I. Emprego do SERVQUAL na avaliação da qualidade de serviços de tecnologia da informação: uma análise experimental. In: ENCONTRO NACIONAL DE ENGENHARIA DE PRODUÇÃO, 26., 
2006, Fortaleza. Anais... Fortaleza: Associação Brasileira de Engenharia de Produção, 2006. p. 1-8.

FREITAS, A. L. P.; MORAIS, A. S. C. Avaliação de serviços de uma agência bancária segundo a percepção de seus clientes. In: ENCONTRO NACIONAL DE ENGENHARIA DE PRODUÇÃO, 28., 2008, Rio de Janeiro. Anais... Rio de Janeiro: Associação Brasileira de Engenharia de Produção, 2008a. p. 1-14.

. Avaliando os serviços de uma agência bancária. Revista Banas Qualidade, p. 20-32, mar. 2008b.

GONÇALVES, T. J. M.; FREITAS, A. L. P. Emprego de um sistema web para aplicação de questionários na avaliação da qualidade em serviços. Revista Ingepro, Santa Maria, v. 2, n. 1, p. 108-120, jan. 2010a.

. Utilização de um modelo SERVPERF para avaliação da qualidade em laboratórios de informática através da aplicação de questionários via web. In: ENCONTRO NACIONAL DE ENGENHARIA DE PRODUÇÃO, 30., 2010, São Carlos. Anais... São Carlos: Associação Brasileira de Engenharia de Produção, 2010b. p. 1-12. . Utilização do sistema SADE na avaliação da qualidade dos serviços prestados em um laboratório de informática: Um estudo de caso em uma universidade estadual. In: ENCONTRO NACIONAL DE ENGENHARIA DE PRODUÇÃO, 29., 2009, Salvador. Anais... Salvador: Associação Brasileira de Engenharia de Produção, 2009. p. 1-12.

GRÖNROOS, C. Marketing, gerenciamento e serviços: A competição por serviços na hora da verdade. 5. ed. Rio de Janeiro: Campus, 1993.

KOTLER, P.; ARMSTRONG, G. Princípios de marketing. 7. ed. Rio de Janeiro: Prentice Hall, 1998.

LOVELOCK, C.; WRIGHT, L. Serviços: marketing e gestão. 3. ed. São Paulo: Saraiva, 2003.

MAGALHÃES, R. C. S. B.; GARCIA, L. S. Lan House: Espaço privado no público. Perspectivas Online, v. 5, n. 2, p. 24-33, 2008.

OLIVER, R. L. A cognitive model of the antecedents and consequences of satisfaction decisions. Journal of Marketing Research, Chicago, v. 17, n. 4, p. 460-469, nov. 1980. . Measurement and evaluation of satisfaction process in retail settings. Journal of Retailing, New York University, v. 57, n. 3, p. 25-48, fall 1981. 
PARASURAMAN, A.; BERRY, L. L.; ZEITHAML, V. A. An empirical examination of relationships in an extended service quality model. Cambridge: Marketing Science Institute, 1990.

. Perceived service quality as a custom based performance measure: an empirical examination of organizational barrier using an extended service quality model. Human Resource Management, v. 30, n. 3, p. 41-50, 1991.

PARASURAMAN, A.; ZEITHAML, V. A.; BERRY, L. L. A conceptual model of service quality and its implications for future research. Journal of Marketing, v. 49, p. 41-50, fall 1985.

. SERVQUAL: A multiple-item scale for measuring consumer perceptions of service quality. Journal of Retailing, New York University, v. 64, n. 1, p. 12-40, spring 1988.

. Refinement and reassessment of the SERVQUAL scale. Journal of Retailing, New York University, v. 67, n. 4, p. 420-450, 1991.

PORTER, M. E. Estratégia competitiva: Técnicas para análise da indústria e da concorrência. 8. ed. Rio de Janeiro: Campus, 1999.

SCHNEIDER, B.; WHITE, S. Service quality: Research perspectives. California: Sage Publications, 2004.

TORRETA, A. Mergulho na base da pirâmide. São Paulo: Saraiva, 2009.

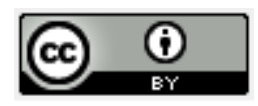

Artigo recebido em 26/11/2010 e aceito para publicação em 29/11/2011. 


\section{APÊNDICE 1: QUESTIONÁRIO APLICADO}

\section{Avaliação da Lan House CyberLink}

Esta pesquisa tem como objetivo avaliar a qualidade dos serviços prestados pela Lan House CyberLink a partir da captação da percepção de seus usuários. Indique o seu Grau de Concordância em relação a cada um dos critérios apresentados abaixo. Utilize a seguinte escala:

(1) Discordo Totalmente

( 2 ) Discordo

( 3 ) Neutro

( 4 ) Concordo

( 5 ) Concordo Totalmente

Marque a opção "Não se Aplica" caso não se julgue com conhecimento suficiente para responder determinado critério.

\begin{tabular}{|c|c|c|c|c|c|c|c|c|c|c|c|c|}
\hline$\overline{N^{\circ}}$ & Item & \multicolumn{5}{|c|}{ Expectativa } & \multicolumn{5}{|c|}{ Desempenho } & \\
\hline 1 & O ambiente é limpo. & 1 & 2 & 3 & 4 & 5 & 1 & 2 & 3 & 4 & 5 & Não se aplica \\
\hline 2 & O ambiente é ventilado. & 1 & 2 & 3 & 4 & 5 & 1 & 2 & 3 & 4 & 5 & Não se aplica \\
\hline 3 & O ambiente é iluminado. & 1 & 2 & 3 & 4 & 5 & 1 & 2 & 3 & 4 & 5 & Não se aplica \\
\hline 4 & $\begin{array}{l}\text { Os funcionários são bem vestidos e } \\
\text { asseados. }\end{array}$ & 1 & 2 & 3 & 4 & 5 & 1 & 2 & 3 & 4 & 5 & Não se aplica \\
\hline 5 & $\begin{array}{l}\text { Velocidade de acesso à Internet é } \\
\text { alta. }\end{array}$ & 1 & 2 & 3 & 4 & 5 & 1 & 2 & 3 & 4 & 5 & Não se aplica \\
\hline 6 & $\begin{array}{l}\text { Desempenho dos computadores é } \\
\text { adequado. }\end{array}$ & 1 & 2 & 3 & 4 & 5 & 1 & 2 & 3 & 4 & 5 & Não se aplica \\
\hline 7 & Softwares instalados são adequados. & 1 & 2 & 3 & 4 & 5 & 1 & 2 & 3 & 4 & 5 & Não se aplica \\
\hline 8 & $\begin{array}{l}\text { Os computadores funcionam } \\
\text { corretamente. }\end{array}$ & 1 & 2 & 3 & 4 & 5 & 1 & 2 & 3 & 4 & 5 & Não se aplica \\
\hline
\end{tabular}

Revista Produção Online, Florianópolis, SC, v.12, n. 1, p. 248-268, jan./mar. 2012. 


\begin{tabular}{|c|c|c|c|c|c|c|c|c|c|c|c|c|}
\hline 9 & $\begin{array}{l}\text { Existe segurança contra a } \\
\text { transmissão de malwares. }\end{array}$ & 1 & 2 & 3 & 4 & 5 & 1 & 2 & 3 & 4 & 5 & Não se aplica \\
\hline 10 & Funciona nos horários divulgados. & 1 & 2 & 3 & 4 & 5 & 1 & 2 & 3 & 4 & 5 & Não se aplica \\
\hline 11 & $\begin{array}{l}\text { O serviço é fornecido assim que } \\
\text { solicitado. }\end{array}$ & 1 & 2 & 3 & 4 & 5 & 1 & 2 & 3 & 4 & 5 & Não se aplica \\
\hline 12 & $\begin{array}{l}\text { Os funcionários são dispostos a } \\
\text { ajudar os clientes. }\end{array}$ & 1 & 2 & 3 & 4 & 5 & 1 & 2 & 3 & 4 & 5 & Não se aplica \\
\hline 13 & Os Funcionários são educados. & 1 & 2 & 3 & 4 & 5 & 1 & 2 & 3 & 4 & 5 & Não se aplica \\
\hline 14 & $\begin{array}{l}\text { Existe suporte adequado para o uso } \\
\text { dos equipamentos. }\end{array}$ & 1 & 2 & 3 & 4 & 5 & 1 & 2 & 3 & 4 & 5 & Não se aplica \\
\hline 15 & $\begin{array}{l}\text { Os funcionários fornecem atenção } \\
\text { individual. }\end{array}$ & 1 & 2 & 3 & 4 & 5 & 1 & 2 & 3 & 4 & 5 & Não se aplica \\
\hline 16 & $\begin{array}{l}\text { O horário de funcionamento é } \\
\text { conveniente. }\end{array}$ & 1 & 2 & 3 & 4 & 5 & 1 & 2 & 3 & 4 & 5 & Não se aplica \\
\hline \multicolumn{13}{|c|}{ Comentários, críticas e sugestões: } \\
\hline
\end{tabular}

Fonte: Elaborado pelos autores

Revista Produção Online, Florianópolis, SC, v.12, n. 1, p. 248-268, jan./mar. 2012. 